\title{
Design and Analysis of Printed Yagi-Uda Antenna and Two-Element Array for WLAN Applications
}

\author{
Cai Run-Nan, ${ }^{1}$ Yang Ming-Chuan, ${ }^{1}$ Lin Shu, ${ }^{2}$ Zhang Xing-Qi, ${ }^{1}$ \\ Zhang Xin-Yue, ${ }^{1}$ and Liu Xiao-Feng ${ }^{1}$ \\ ${ }^{1}$ Communication Research Center, Harbin Institute of Technology, Yikuang Street 2, Harbin 150080, China \\ ${ }^{2}$ Electronic Science and Technology Post-Doctoral Research Center, Harbin Institute of Technology, Yikuang Street 2, \\ Harbin 150080, China
}

Correspondence should be addressed to Yang Ming-Chuan, mcyang@hit.edu.cn

Received 5 June 2012; Accepted 31 August 2012

Academic Editor: Ahmed A. Kishk

Copyright () 2012 Cai Run-Nan et al. This is an open access article distributed under the Creative Commons Attribution License, which permits unrestricted use, distribution, and reproduction in any medium, provided the original work is properly cited.

\begin{abstract}
A printed director antenna with compact structure is proposed. The antenna is fed by a balanced microstrip-slotline and makes good use of space to reduce feeding network area and the size of antenna. According to the simulation results of CST MICROWAVE STUDIO software, broadband characteristics and directional radiation properties of the antenna are explained. The operating bandwidth is $1.8 \mathrm{GHz}-3.5 \mathrm{GHz}$ with reflection coefficient less than $-10 \mathrm{~dB}$. Antenna gain in band can achieve $4.5-6.8 \mathrm{dBi}$, and the overall size of antenna is smaller than $0.34 \lambda_{0} \times 0.58 \lambda_{0}$. Then the antenna is developed to a two-element antenna array, working frequency and relative bandwidth of which are $2.15-2.87 \mathrm{GHz}$ and $28.7 \%$, respectively. Compared with antenna unit, the gain of the antenna array has increased by $2 \mathrm{~dB}$. Thus the proposed antenna has characteristics of compact structure, relatively small size, and wideband, and it can be widely used in PCS/UMTS/WLAN/ WiMAX fields.
\end{abstract}

\section{Introduction}

The extensive and intensive growth of wireless communication systems has led to an increasing demand for antennas that can be printed on a substrate. And printed antenna has wide applications because of light weight, low profile, small volume, easiness to integrate with communication system, and so forth.

In 1998, printed quasi-Yagi-Uda antenna was first proposed by Qian and others [1], and it is still an important issue in today's research activities. This quasi-Yagi-Uda antenna combines the excellent radiation properties of the Yagi-Uda antenna with the versatility of the microstrip technique. The balanced to unbalanced transition design determines the broadband characteristics of Yagi-Uda antenna. Microstripto-coplanar-strip (MS-to-CPS) transition is designed in [1], and it reaches $17 \%$ relative bandwidth. Besides, reference [2] proposes a coplanar waveguide-coplanar strip (CPWto-CPS) transition, which can excite the odd-mode electric field at the CPS line though the CPW feeding line. And at the same time, the antenna can work at multiple frequency points. The odd-mode at the CPS line can also be excited by providing $180^{\circ}$ phase delay [3]. These years, the feeding structure of the Yagi-Uda antenna is also directly realized by a microstrip line or a simple CPW without any transition structure $[4,5]$. For the above research, a common character is that large ground plates are used as reflectors. As a result, these antennas usually have large size and are unbeneficial for antenna miniaturization as well as array composing. If feeding structure without large ground plate can be designed, the size and complexity can be greatly reduced [6].

On the other hand, printed Yagi-Uda antenna array can also be widely applied in communication systems. First, Yagi-Uda antenna array has the potential to be an attractive solution to design phased-array and multiple-beam antennas $[7,8]$. Second, Yagi-Uda antenna array can be designed as microstrip patch form and could be applied into mobile satellite (MSAT) field $[9,10]$. Third, although most wireless local area network (WLAN) applications utilize standard omnidirectional antennas, directional antennas, such as 


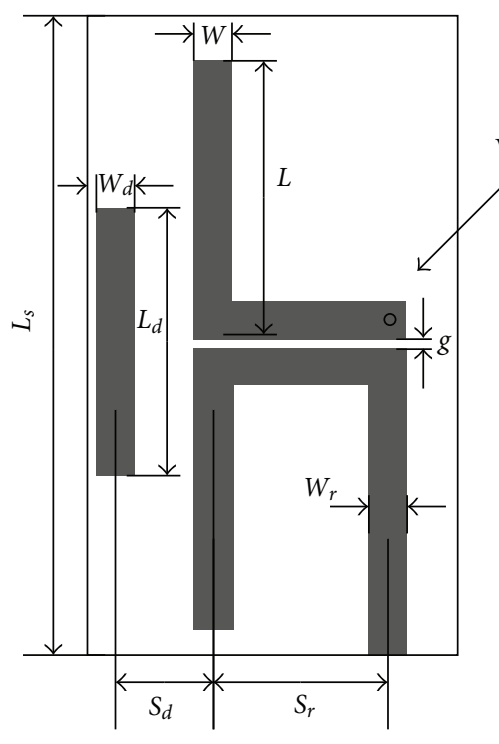

(a)

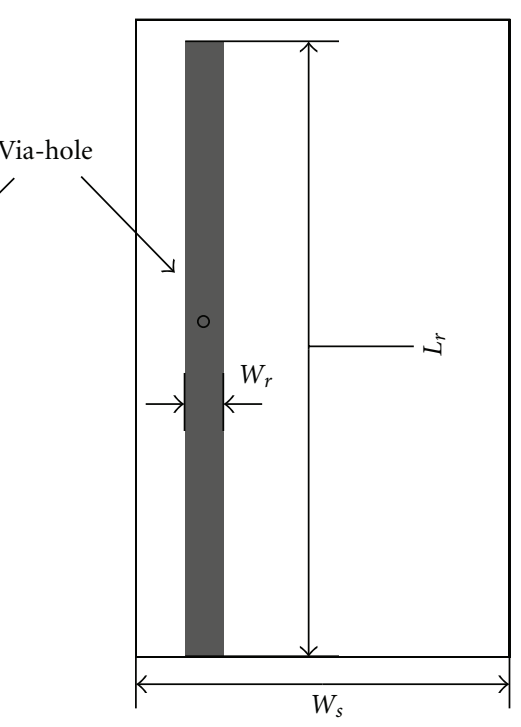

(b)

FIGURE 1: Structure of printed Yagi-Uda antenna unit. (a) Front view and (b) back view. ( $W_{d}=4 \mathrm{~mm}, W_{r}=4 \mathrm{~mm}, W=4 \mathrm{~mm}, S_{d}=10 \mathrm{~mm}$, $\left.S_{r}=18 \mathrm{~mm}, L_{d}=28 \mathrm{~mm}, L_{r}=64 \mathrm{~mm}, L=29 \mathrm{~mm}, L_{s}=66 \mathrm{~mm}, W_{s}=38 \mathrm{~mm}, g=0.8 \mathrm{~mm}\right)$.

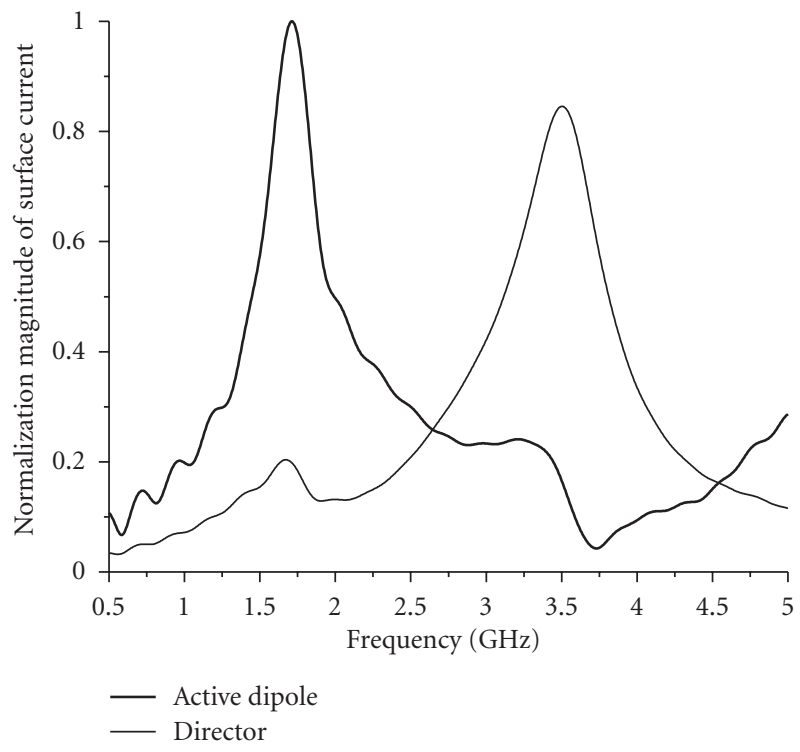

FIGURE 2: Simulated results of current amplitude at the middle of both the director and active dipole.

quasi-Yagi-Uda arrays, have been employed to suppress unwanted radio frequency emission and/or interference in required directions. However, miniature printed Yagi-Uda antenna array covering WLAN range is very rare. And the two main parts in designing printed Yagi-Uda antenna array are separately feeding network design and coupling effect suppression $[11,12]$.

A simple and compact printed Yagi-Uda antenna fed by balanced microstrip-slotline and its array are presented based on $[13,14]$. Balanced microstrip line replaces traditional

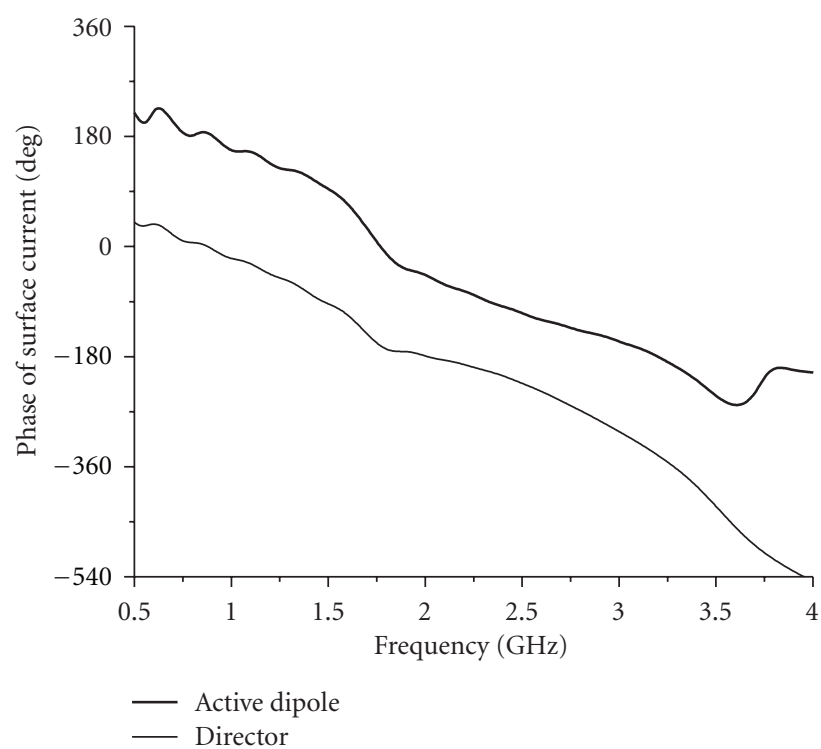

Figure 3: Simulated results of antenna surface current phase.

ground plate to be the reflector of the Yagi-Uda antenna. And the characteristics of compact size and broad band are achieved. The balanced to unbalanced transition is designed through a quasimicrostrip structure. The final proposed Yagi-Uda antenna unit can work at $1.8 \mathrm{GHz}-3.5 \mathrm{GHz}$ with reflection coefficient less than $-10 \mathrm{~dB}$. The ratio bandwidth is $1.94: 1$, and stable gain in band can achieve $4.5-6.8 \mathrm{dBi}$. The overall size of the proposed antenna unit is smaller than $0.34 \lambda_{0} \times 0.58 \lambda_{0}$. Then a two-element Yagi-Uda antenna array with $28.7 \%$ relative bandwidth and $8.5 \mathrm{dBi}$ gain (maximum) 


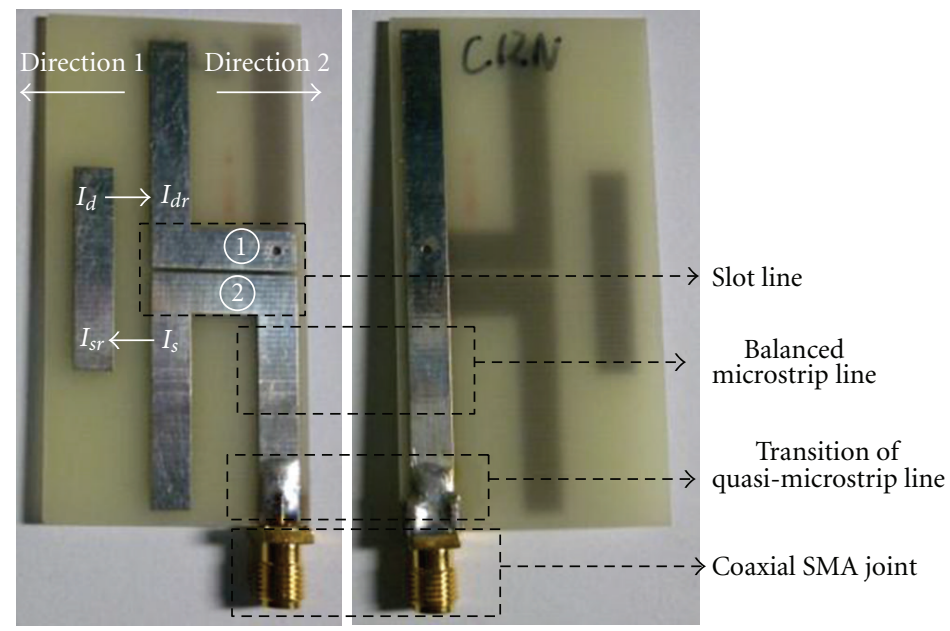

FIGURE 4: Feeding structure of the proposed printed Yagi-Uda antenna unit.

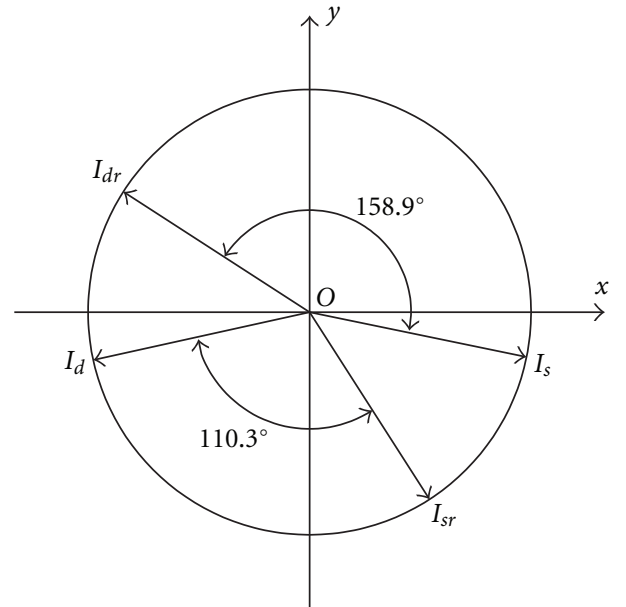

(a) $1.8 \mathrm{GHz}, I_{d}=0.18 \angle-167.6^{\circ}, I_{s}=1.00 \angle-11.9^{\circ}$, $I_{s r}=1.00 \angle-57.3^{\circ}, I_{d r}=0.18 \angle-213^{\circ}$

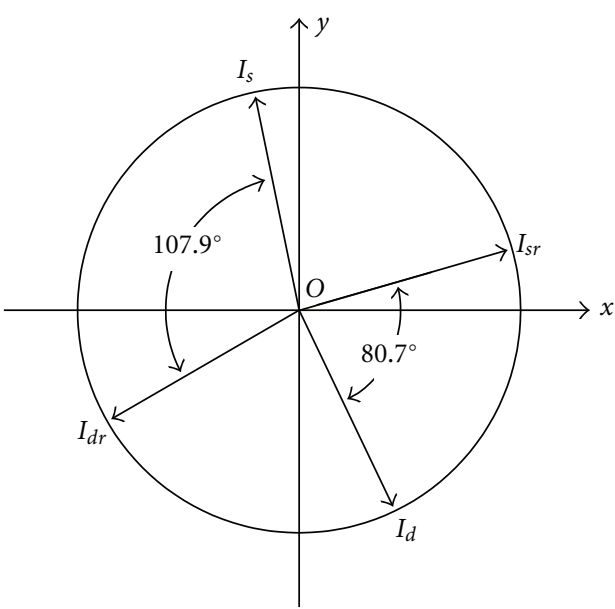

(b) $3.4 \mathrm{GHz} I_{d}=1.00 \angle-64.8^{\circ}, I_{s}=0.22 \angle$ $101.6^{\circ}, I_{s r}=0.22 \angle 15.9^{\circ}, I_{d r}=1.00 \angle-150.5^{\circ}$

FIGURE 5: Equivalent radiation current vector diagram of the antenna.

is presented. It can cover WLAN range and has potential use in broadband mobile applications.

\section{Design and Experiment of Yagi-Uda Antenna Fed by Balanced Microstrip Line}

2.1. Printed Yagi-Uda Antenna Unit Structure. The proposed Yagi-Uda antenna unit has a structure of double PCB, and the relative position between the metal shapes printed on the top and bottom of the dielectric substrate is shown in Figure 1. The whole antenna can be divided into three parts: on the top, the dark area in the left end is director of metal-strip form, the part in the middle is the active dipole, and the rest is feeding line. The feeding line is a structure transformed from balanced microstrip line to slot line, and it consists of two metal-strip branches printed on the top and bottom surfaces. The metal strip on the top is directly connected with one branch of the dipole through the slot line, while the metal strip on the bottom is connected to the other branch of the dipole through a via-hole and the slot line. At the same time, on the bottom the balanced microstrip line is upward prolonged from the via-hole compared to the top bottom, which acts as a reflector and can adjust the bandwidth.

2.2. Principle of the Printed Yagi-Uda Antenna Unit. The simulated current amplitude results of the middle part of the director and the middle part of the active dipole are shown in Figure 2. The director current peak is corresponding to $3.5 \mathrm{GHz}$, and the resonant frequency of active dipole is corresponding to $1.7 \mathrm{GHz}$. Therefore, for the whole antenna composed of two radiation parts, the mutual coupling of two radiation parts will finally form a relatively wide band $(1.7 \mathrm{GHz}-3.5 \mathrm{GHz})$. According to the simulation results, the proposed antenna is a kind of broadband antenna with working bandwidth of $1.8 \mathrm{GHz}-3.4 \mathrm{GHz}$ and relative bandwidth of $61.5 \%$ (ratio bandwidth $1.89: 1$ ). The broadband 


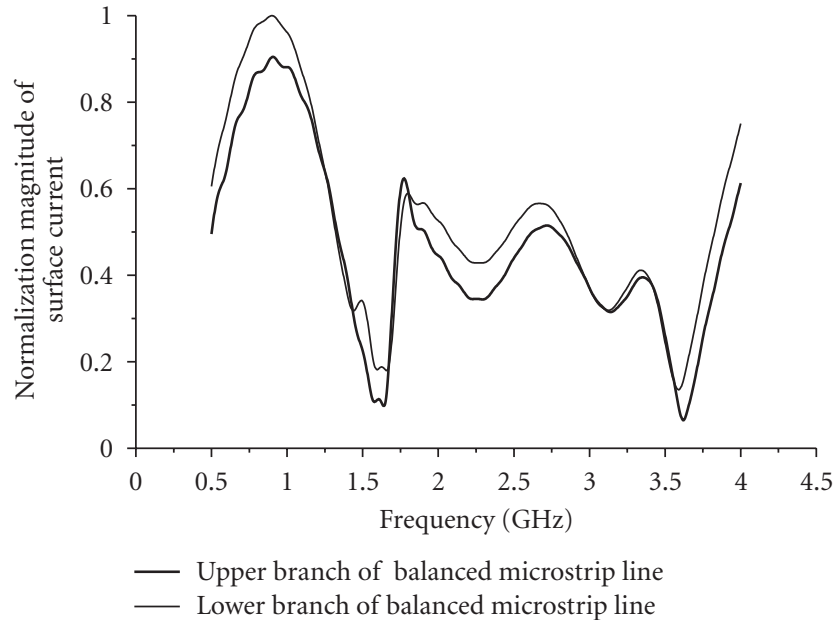

(a) Amplitude

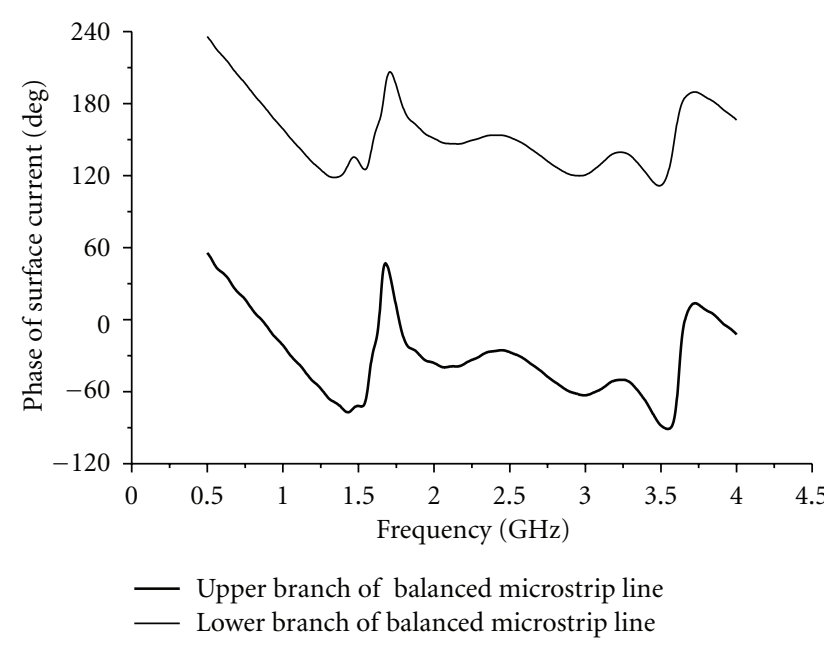

(b) Phase

FIGURE 6: Simulated results of surface current on the terminal of the balanced microstrip line.

mechanism is due to double resonance phenomenon of the two radiation parts, which is the result of double frequency coupling, essentially.

The simulated results of surface current phase of the two radiation parts (shown in Figure 3 ) indicate that in a relatively wide bandwidth, director current phase falls behind the phase of active dipole current. And in the frequency range of $1.8-3.4 \mathrm{GHz}$ the phase difference is $90^{\circ}-$ $120^{\circ}$, which determines the directionality of the radiation in the frequency band. In Figure 1, the central distance between the active dipole and director is $S_{d}=10 \mathrm{~mm} . \Delta \varphi$ is the spatial phase shift generated after current vector travels $S_{d}$ distance. And the formula for $\Delta \varphi$ is as follows:

$$
\Delta \phi=k_{g} S_{d}=25.2 f(\text { deg. }),
$$

where $k_{g}$ is the wave number in the medium. And unit of $f$ is $\mathrm{GHz}$. Then $\Delta \varphi$ is $45.4^{\circ}$ at $1.8 \mathrm{GHz}$, and $85.7^{\circ}$ at $3.4 \mathrm{GHz}$. Besides, the radiation field caused by antenna current is

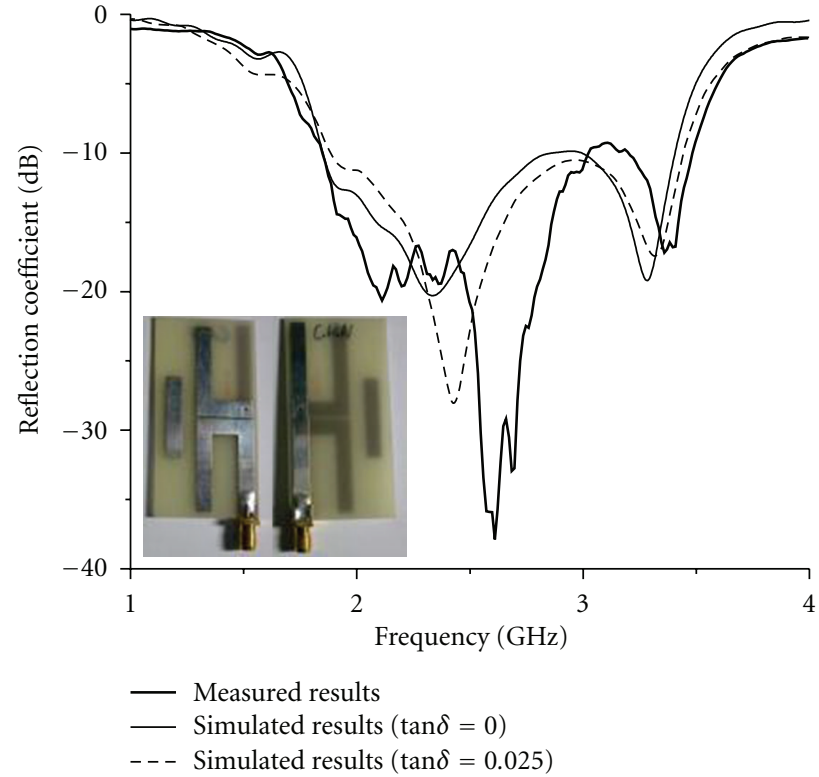

FIGURE 7: Simulated and experimental reflection coefficient results of the printed Yagi-Uda antenna unit.

analyzed through vector diagram. As shown in Figures 4 and 5 , the four vectors drawn in complex plane are current vector of director $I_{d}$ and current vector of active dipole $I_{s}, I_{d r}$ (vector sum of current vector of director and additional spatial phase shift $\Delta \varphi$ ), and $I_{s r}$ (vector sum of current vector of active dipole and additional spatial phase shift $\Delta \varphi$ ). In addition, angle between $I_{d}$ and $I_{s r}$ is smaller than angle between $I_{d r}$ and $I_{s}$ at the boundary frequency points of $1.8 \mathrm{GHz}$ and $3.4 \mathrm{GHz}$, which can illuminate that current radiation field will be enhanced in direction 1, and the two currents in direction 2 can cause approximate reversed-phase superposition, thus the field distribution will be decreased. It can be concluded that the proposed antenna has characteristics of directional radiation in a wide bandwidth.

2.3. Feeding Structure of the Printed Yagi-Uda Antenna Unit. The feeding structure of actual antenna is presented in Figure 4. The whole antenna can be divided into four parts, the coaxial line part, the quasimicrostrip line part, the balanced microstrip line part, and the slot line part. On one side, the feeding line is connected to the down branch of the dipole directly; on the other side, the feeding line is connected to the up branch of the dipole through a via-hole. The balanced microstrip line transits to the coaxial SMA joint through a section of quasimicrostrip line, which can be regarded as a broadband Balun structure. The simulated results in Figure 6 show that there is a little difference between the amplitudes at port (1) and port (2) (indicated in Figure 4). Besides, their phase difference is about $180^{\circ}$. Thus through the quasi-balanced-microstrip line transition, slot line can achieve the balanced feeding to the dipole.

On the other hand, in most cases, the dipole will be printed on both sides of the dielectric substrate, which will make the two branches of the dipole noncoaxial and 


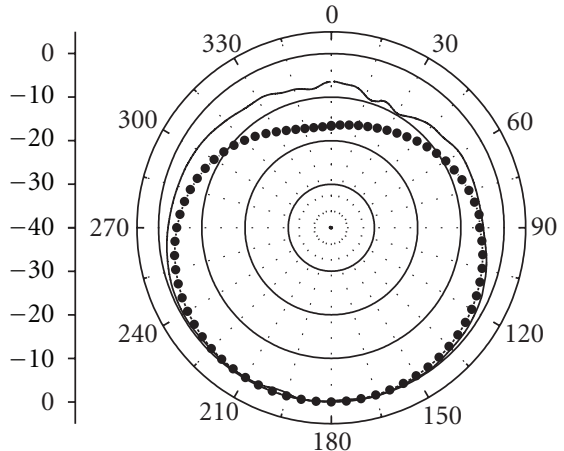

(a) $1.8 \mathrm{GHz}, \mathrm{H}$ plane

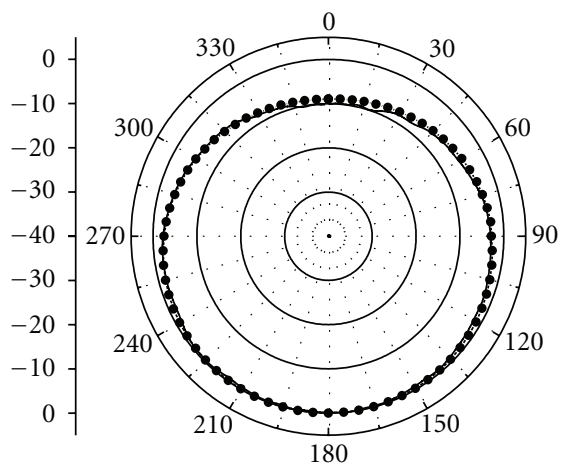

(c) $2.4 \mathrm{GHz}, \mathrm{H}$ plane

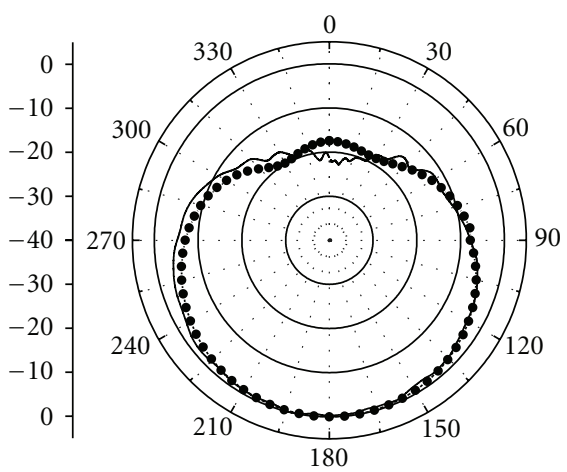

$-\bullet$ Simulated results
- Measured results

(e) $3.5 \mathrm{GHz}, \mathrm{H}$ plane

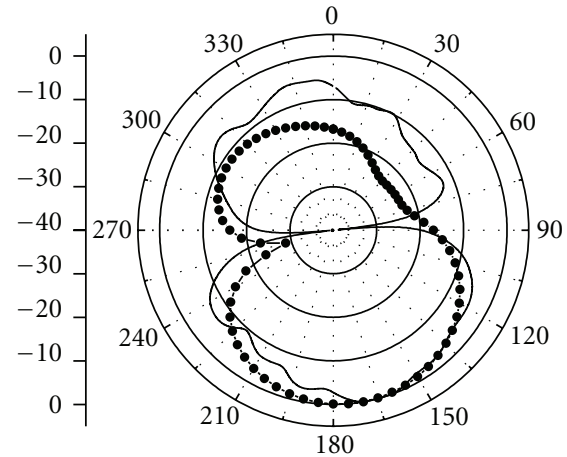

(b) $1.8 \mathrm{GHz}$, E plane

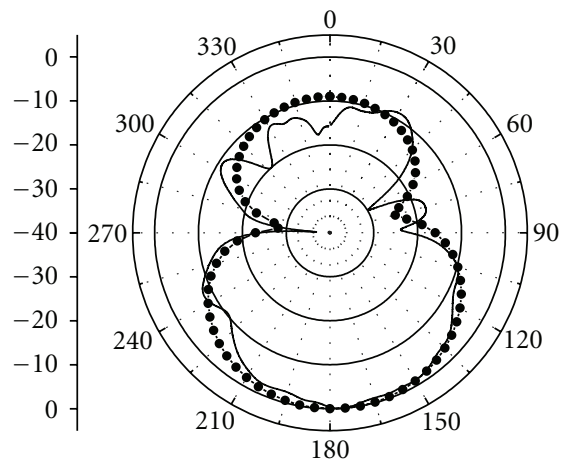

(d) $2.4 \mathrm{GHz}$, E plane
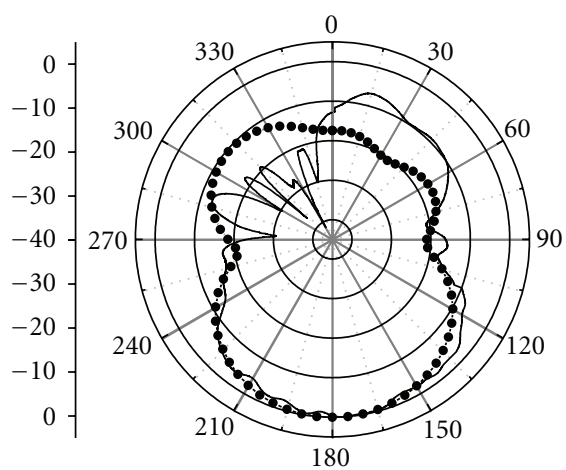

- - Simulated results

- Measured results

(f) $3.5 \mathrm{GHz}$, E plane

FIGURE 8: Simulated and experimental radiation patterns of the printed Yagi-Uda antenna unit.

destroy the symmetry of the radiation pattern. In case of this condition, the two branches of the dipole are printed on the same side of the dielectric substrate achieved through a via-hole design. As the feeding vertexes of the two dipoles have a short distance and the dielectric substrate is very thin (merely $1.5 \mathrm{~mm}$, equivalent to $1.7 \%$ of the minimum radiation wavelength), the engendered phase shift can be neglected.

2.4. Experimental Results of the Printed Yagi-Uda Antenna Unit. Prototype of the proposed antenna has been measured in the anechoic chamber using the Agilent E8363B vector
TABLe 1: The simulated and experimental results of the printed Yagi-Uda antenna unit gain.

\begin{tabular}{lllll}
\hline & Frequency $(\mathrm{GHz})$ & 1.8 & 2.4 & 3.5 \\
\hline \multirow{3}{*}{ Gain $(\mathrm{dBi})$} & Simulated $(\tan \delta=0)$ & 5.4 & 5.0 & 7.2 \\
& Simulated $(\tan \delta=0.025)$ & 4.4 & 4.5 & 5.5 \\
& Measured & 4.5 & 4.8 & 6.8 \\
\hline
\end{tabular}

network analyzer. The measuring reflection coefficient and normalized radiation pattern are presented in Figures 7 and 8. The proposed antenna can well operate in the bandwidth 


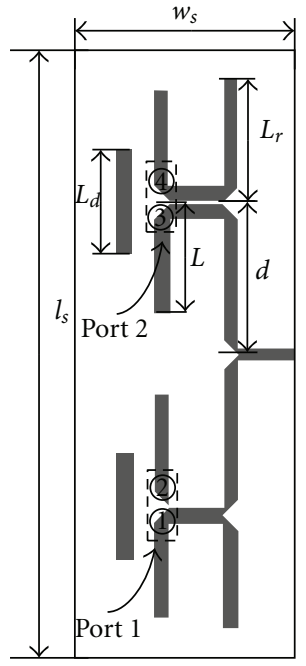

(a)

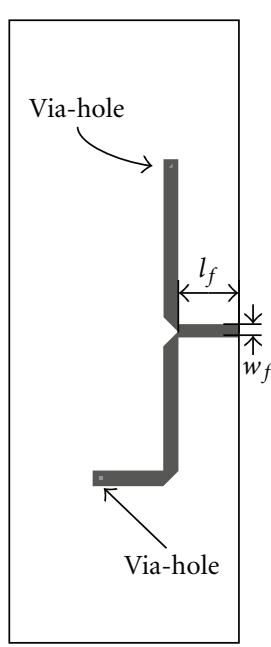

(b)

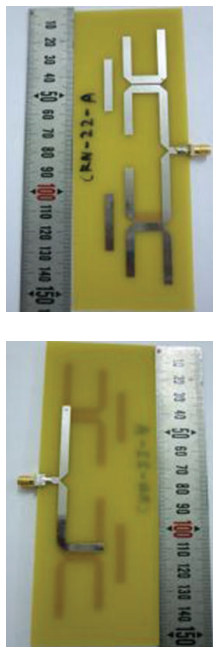

(c)

Figure 9: Sketch of the proposed Yagi-Uda antenna array with associated geometrical parameters. (a) Front view. (b) Back view. (c) The prototype of the printed Yagi-Uda antenna array. $\left(L_{d}=28 \mathrm{~mm}, L_{r}=36 \mathrm{~mm}, L=26 \mathrm{~mm}, S_{d}=10 \mathrm{~mm}, S_{r}=18 \mathrm{~mm}, d=35 \mathrm{~mm}, w_{s}=\right.$ $\left.58 \mathrm{~mm}, l_{s}=155 \mathrm{~mm}, w_{f}=3 \mathrm{~mm}, l_{f}=10 \mathrm{~mm}\right)$.

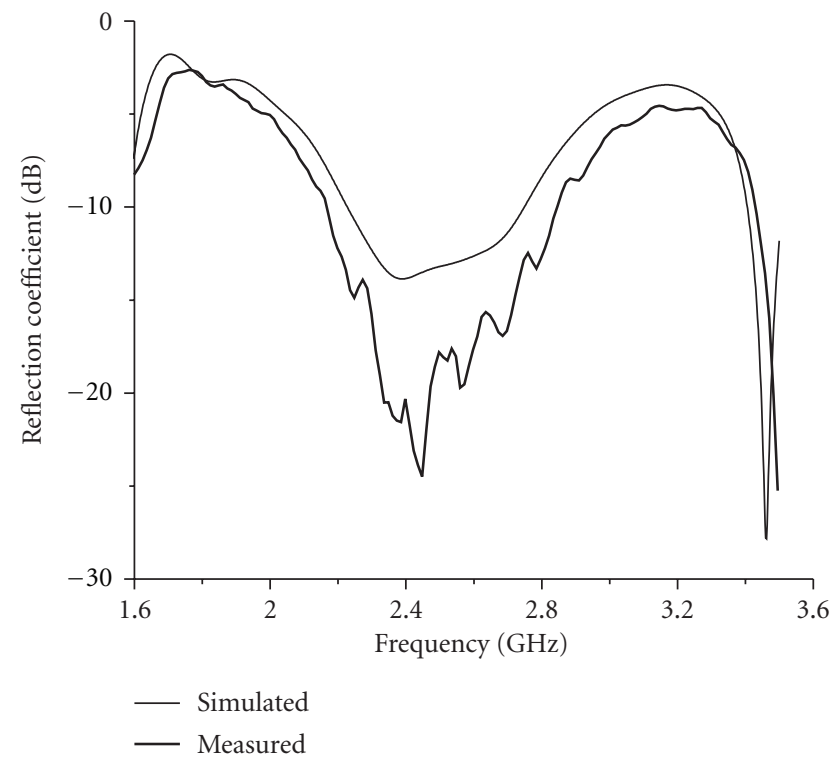

FIGURE 10: Simulated and experimental reflection coefficient results of the proposed Yagi-Uda antenna array.

TABLE 2: The simulated and experimental results of the printed Yagi-Uda antenna array gain.

\begin{tabular}{lllll}
\hline Frequency $(\mathrm{GHz})$ & 2.2 & 2.4 & 2.6 & 2.8 \\
\hline Simulated gain $(\mathrm{dBi})$ & 6.6 & 7.3 & 8.0 & 8.8 \\
Experimental gain $(\mathrm{dBi})$ & 6.4 & 7.1 & 7.7 & 8.5 \\
\hline
\end{tabular}

of $1.8-3.5 \mathrm{GHz}$ (relative bandwidth $64.2 \%$, ratio bandwidth $1.94: 1$ ) with reflection coefficient less than $-10 \mathrm{~dB}$, which is in accordance with the simulated results. Because of the loss of the applied FR4 resin epoxy medium, the measured results of reflection coefficient (in the bandwidth of $2.5-3.0 \mathrm{GHz}$ ) have much difference with the ideal simulated results. If the ideal medium is changed to lossy medium (loss tangent is 0.025), then the simulated results match the experimental results perfectly well. And it is the same reason for the decrease of antenna gain and radiation pattern difference. The tested results of antenna gain are listed in Table 1, which are consistent with the simulated results of loss tangent.

\section{Two-Element Printed Yagi-Uda Antenna Array}

In order to improve the gain, we design a two-element array composed of two proposed printed Yagi-Uda antenna units, and the prototype of such antenna array is shown in Figure 9. The instant polarity method is used to design the feeding network of the array. That is, assume the polarity of the upper strip of the balanced microstrip line is positive, and the polarity of the underneath strip is negative, thus at port 1 , the polarity of (1) terminal is positive, and so is terminal (3) which connects with the upper strip. At the same time, the polarity is negative for terminal (4) and terminal (2), which connect with underneath strip through a via-hole separately. Thus, the proposed feed structure can enhance antenna gain through radiation field in-phase superposition of the two antenna units.

As shown in Figure 10, the tested results indicate that the proposed antenna array has good broadband performance in 2.15-2.87 GHz with reflection coefficient less than $-10 \mathrm{~dB}$. And it can cover WLAN frequency range. And because of the mutual coupling between antenna elements in the array, the bandwidth of the proposed antenna array is smaller than the antenna unit bandwidth. The simulated and experimental results of normalized radiation pattern 


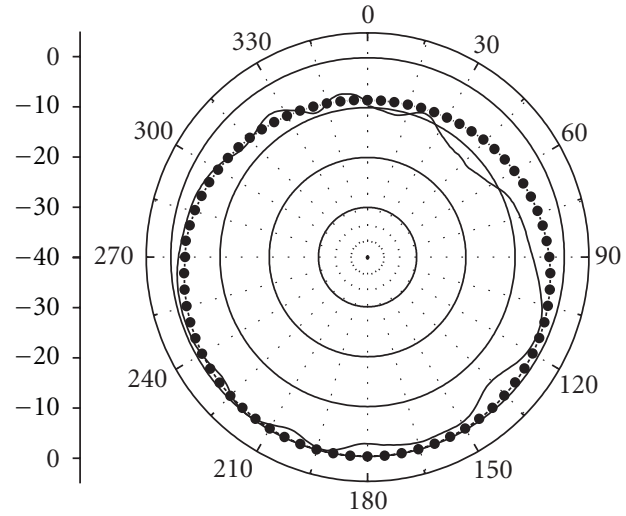

(a) $2.2 \mathrm{GHz}, \mathrm{H}$ plane

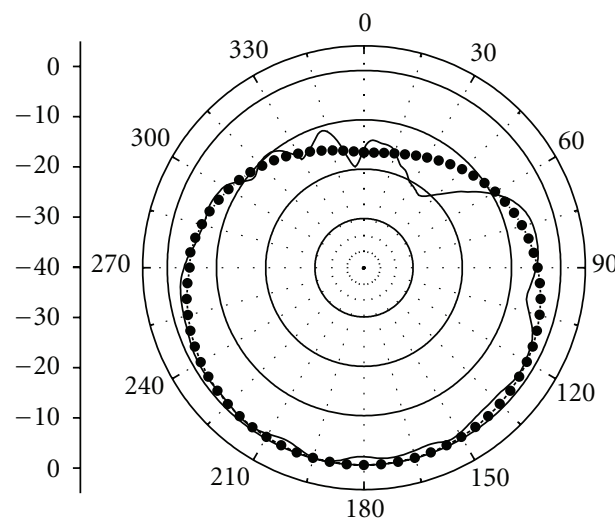

(c) $2.5 \mathrm{GHz}, \mathrm{H}$ plane

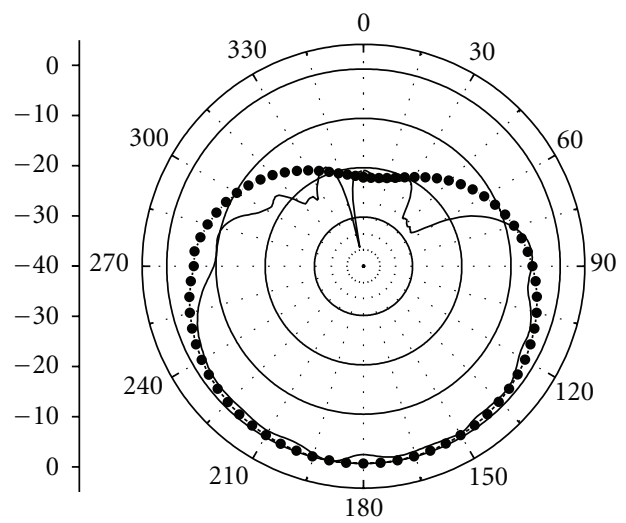

- - Simulated results

(e) $2.8 \mathrm{GHz}, \mathrm{H}$ plane

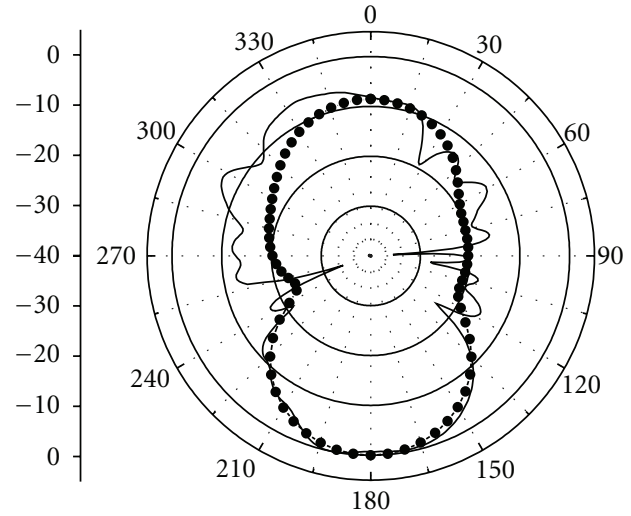

(b) $2.2 \mathrm{GHz}$, E plane

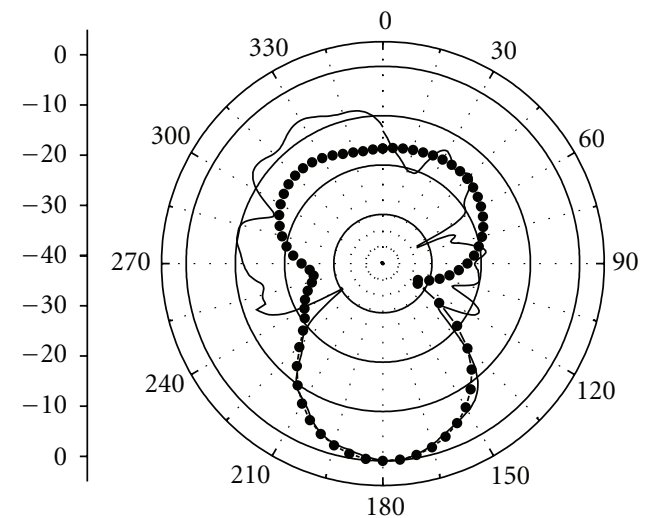

(d) $2.5 \mathrm{GHz}$, E plane

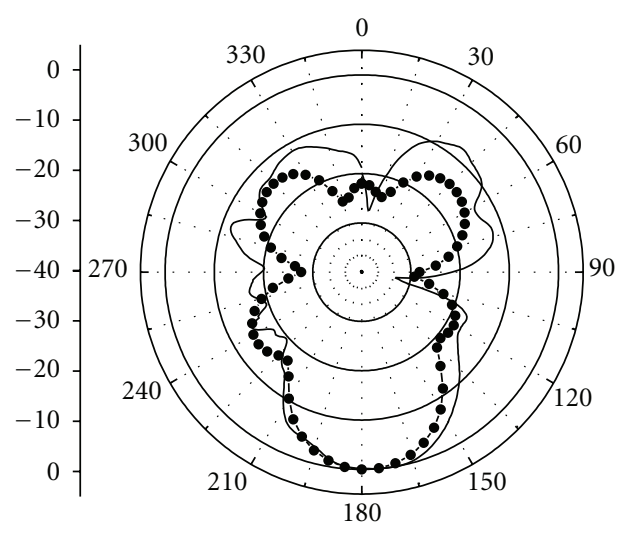

- - Simulated results

- Measured results

(f) $2.8 \mathrm{GHz}$, E plane

FIGURE 11: Simulated and experimental radiation patterns of the printed Yagi-Uda antenna array.

and gain are shown in Figure 11 and Table 2, which indicate that the printed Yagi-Uda antenna array achieves high gain and good directional radiation character. Comparing with the Yagi-Uda antenna unit, the gain of the proposed array has increased by about $2 \mathrm{~dB}$. And the reason for the slight difference between simulated and experimental results lies in the loss of the medium.

\section{Conclusion}

A novel director antenna with compact structure fed by coaxial-quasimicrostrip line-balanced microstrip line-slot line is proposed for the first time, which can achieve balanced feeding in a pretty wide bandwidth. It can be applied to the feeding of the printed Yagi-Uda antenna, and the feeding 
structure size decreases apparently. The designed antenna gain can achieve 4.5-6.8 dBi with relative bandwidth $64.2 \%$, while the biggest size is just 0.58 times of the corresponding wave length. Then we design a two-element array, and the coupling becomes bigger because of the undersize between antenna units. And as a result, antenna bandwidth and gain will degrade. The proposed antenna array can work at 2.15$2.87 \mathrm{GHz}$ with reflection coefficient less than $-10 \mathrm{~dB}$, and antenna gain increases by about $2 \mathrm{~dB}$ compared with antenna unit.

The antenna mentioned in this paper can be printed on low-loss dielectric substrate with higher relative permittivity. Influenced by dielectric, the antenna size will be smaller and the antenna will be easily integrated in microwave circuit. Therefore, this antenna can be widely applied in many fields like PCS/UMTS/WLAN/WiMAX.

\section{Acknowledgments}

This work was supported by the funds from National Natural Science Foundation of China (no. 61101126) and China Postdoctoral Science Foundation (no. 2011M500664).

\section{References}

[1] Y. Qian, W. R. Deal, N. Kaneda, and T. Itoh, "Microstrip-fed quasi-Yagi antenna with broadband characteristics," Electronics Letters, vol. 34, no. 23, pp. 2194-2196, 1998.

[2] Y. Ding, Y. C. Jiao, P. Fei, B. Li, and Q. T. Zhang, "Design of a multiband quasi-Yagi-type antenna with CPW-to-CPS transition," IEEE Antennas and Wireless Propagation Letters, vol. 10, pp. 1120-1123, 2011.

[3] S. J. Wu, C. H. Kang, K. H. Chen, and J. H. Tarng, "A multiband quasi-yagi type antenna," IEEE Transactions on Antennas and Propagation, vol. 58, no. 2, pp. 593-596, 2010.

[4] H. D. Lu, L. M. Si, and Y. Liu, "Compact planar microstripfed quasi-Yagi antenna," Electronics Letters, vol. 48, no. 32, pp. 140-141, 2012.

[5] H. K. Kan, R. B. Waterhouse, A. M. Abbosh, and M. E. Bialkowski, "Simple broadband planar CPW-fed quasi-Yagi antenna," IEEE Antennas and Wireless Propagation Letters, vol. 6, pp. 18-20, 2007.

[6] K. Han, Y. Park, H. Choo, and I. Park, "Broadband CPSfed Yagi-Uda antenna," Electronics Letters, vol. 45, no. 24, pp. 1207-1209, 2009.

[7] Y. - Bai, S. Xiao, M.-C. Tang, Z.-F. Ding, and B.-Z. Wang, "Wide-angle scanning phased array with pattern reconfigurable elements," IEEE Transactions on Antennas and Propagation, vol. 59, no. 11, pp. 4071-4076, 2011.

[8] P. R. Grajek, B. Schoenlinner, and G. M. Rebeiz, "A 24-GHz high-gain Yagi-Uda antenna array," IEEE Transactions on Antennas and Propagation, vol. 52, no. 5, pp. 1257-1261, 2004.

[9] G. R. DeJean, T. T. Thai, S. Nikolaou, and M. M. Tentzeris, "Design and analysis of microstrip bi-yagi and quad-yagi antenna arrays for WLAN applications," IEEE Antennas and Wireless Propagation Letters, vol. 6, pp. 244-248, 2007.

[10] N. Honma, T. Seki, and K. Nishikawa, "Compact planar foursector antenna comprising microstrip Yagi-Uda arrays in a square configuration," IEEE Antennas and Wireless Propagation Letters, vol. 7, pp. 596-598, 2008.

[11] F. Weinmann, "Design, optimization, and validation of a planar nine-element quasi-Yagi antenna array for X-band applications: antenna designer's notebook," IEEE Antennas and Propagation Magazine, vol. 49, no. 2, pp. 89-96, 2007.

[12] N. Nikolic and A. R. Weily, "Compact E-band planar quasiYagi antenna with folded dipole driver," IET Microwaves, Antennas and Propagation, vol. 4, no. 11, pp. 1728-1734, 2010.

[13] C. Run-Nan, L. Shu, H. Guan-Long et al., "Research on a novel Yagi-Uda antenna fed by balanced microstrip line," in Proceedings of the China-Japan Joint Microwave Conference (CJMW'11), pp. 101-104, April 2011.

[14] T. Kouzaki, K. Kimoto, S. Kubota, A. Toya, N. Sasaki, and T. Kikkawa, "Quasi Yagi-Uda antenna array for detecting targets in a dielectric substrate," in Proceedings of the IEEE International Conference on Ultra-Wideband, (ICUWB'09), pp. 759762, September 2009. 

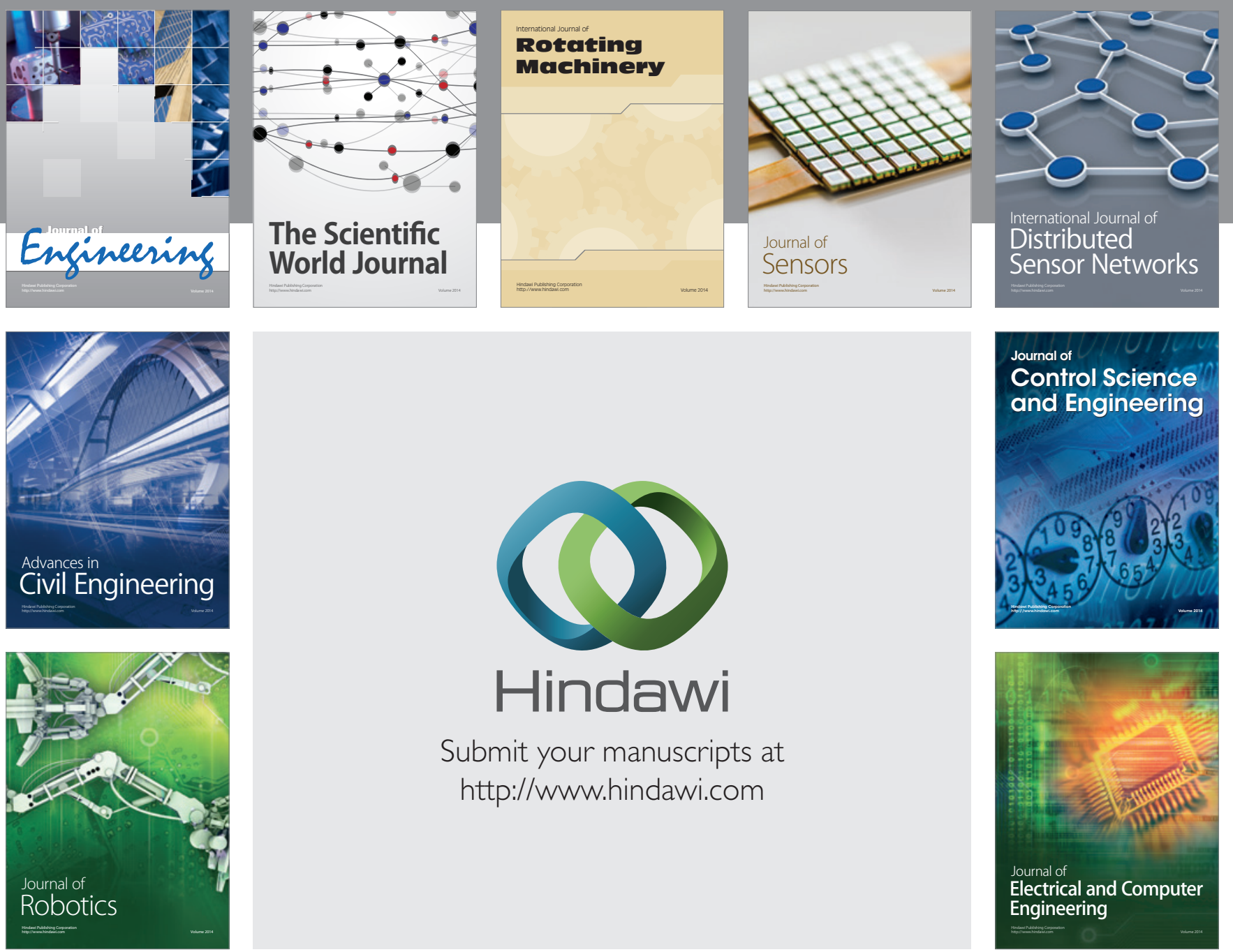

Submit your manuscripts at

http://www.hindawi.com
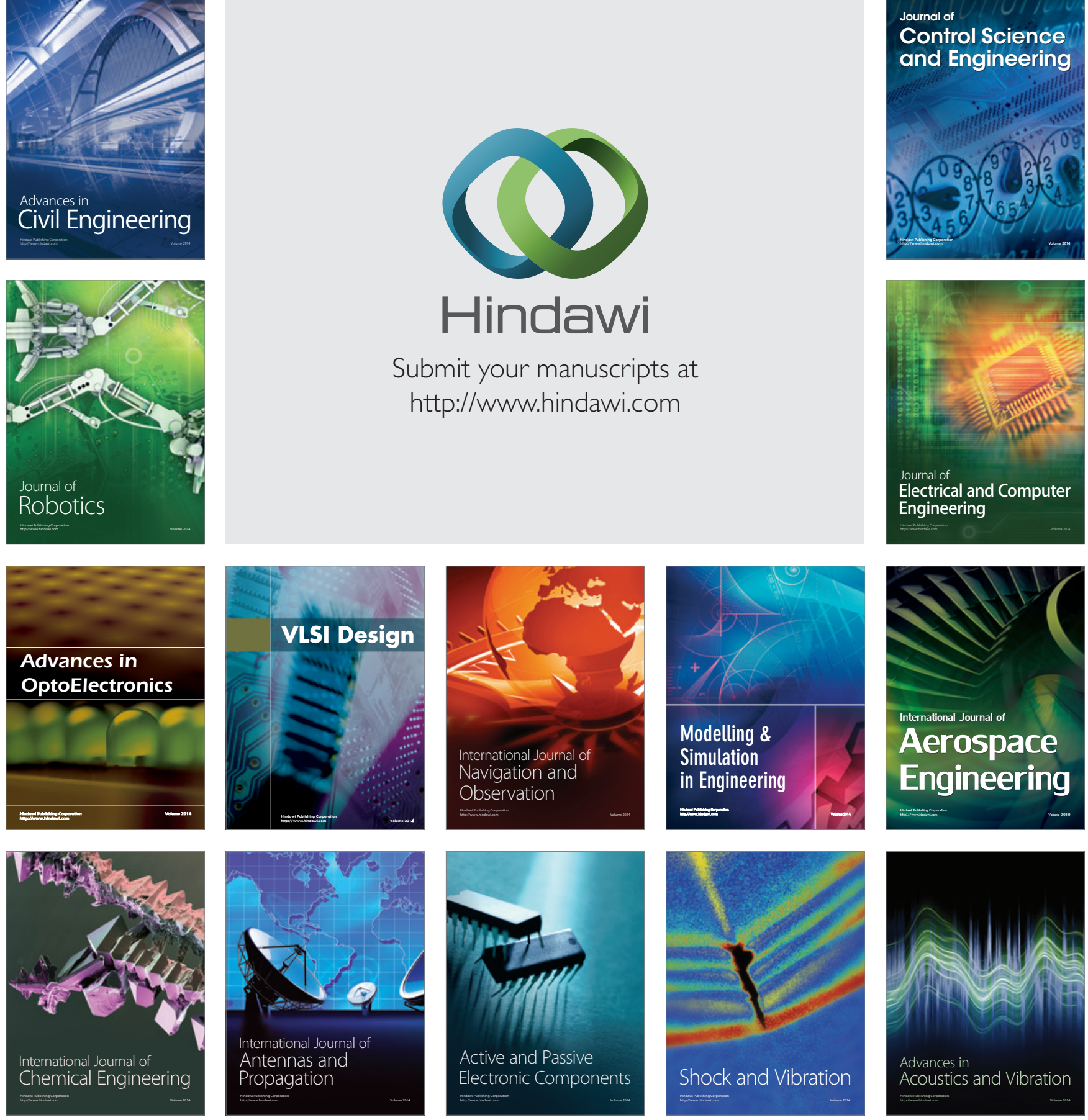